\title{
COMPARING FREQUENCY SCALING EFFICACY ON DIFFERENT MEMORY TECHNOLOGIES
}

\author{
Vaibhav Sundriyal \\ Masha Sosonkina \\ Department of Modeling, Simulation \\ and Visualization Engineering \\ Old Dominion University, Norfolk, USA \\ vsundriy,msosonki@odu.edu
}

\author{
Bryce M. Westheimer \\ Department of Chemistry, \\ Iowa State University, \\ Ames, Iowa, USA \\ westheb@iastate.edu
}

\begin{abstract}
Dual inline memory modules (DIMMs) built with double data rate of the 4th generation (DDR4) synchronous dynamic random access memory (SDRAM) are widely used in current memory components in the high performance computing (HPC) systems. Since modern parallel applications are becoming more data intensive, improvements in memory technology are crucial for sustained high performance. DDR4 provides higher reliability, availability, and serviceability than other DDR memories do so. DDR4 essentially operates at a higher frequency and lower voltage compared with its previous generations. Therefore, for a given application, the memory intensities (measured as a number of memory accesses per micro-operation retired) of DDR3 and DDR4 may differ significantly. In this paper, the difference in memory intensity between DDR3 and DDR4 is explored along with the relative difference in the off-chip time each platform provides to facilitate frequency scaling.
\end{abstract}

\section{INTRODUCTION}

Memory is being considered one of the main bottlenecks while extracting maximum performance from modern supercomputing systems. Most of the current generations systems are being designed with DDR4 SDRAM-based DIMMs. DDR4 SDRAM, an abbreviation for Double Data Rate type 4 Synchronous Dynamic Random Access Memory is the latest member of DDR family of technologies. The DDR4 chips operate at a relatively lower voltage and higher frequency compared to the DDR3 chips along with a larger number of banks as well (JEDEC 2019a, JEDEC 2019b). DDR4 also improves over DDR3 in terms of power consumption.

It is well-established that CPU and the memory subsystem are the major power consumers in a computing system. For example, CPU consumes about $50 \%$ of the total power as was investigated in (Ge, Feng, Song, Chang, Li, and Cameron 2010), considering both static and dynamic power consumption. The current generation of Intel processors provides various P-states for dynamic voltage and frequency scaling (DVFS) and T-states for introducing processor idle cycles (throttling). For example, the Intel "Haswell-EP" microarchitecture provides twelve P-states $f_{p}(1), \ldots, f_{p}(15)$ ranging from $1.2 \mathrm{GHz}$ to $2.3 \mathrm{GHz}$.

Memory intensity of an application can be defined as the number of memory accesses it makes per microoperation retired per second. Performance models proposed in the past (Sundriyal and Sosonkina 2016) which relate application performance to DVFS, depict a strong correlation between memory intensity of an application with the amount of energy savings that can be achieved with DVFS. This can be understood 
from the fact that an application with relatively high memory intensity, tends to have a higher data wait time for the processor and vice versa. This data wait time is exploited through DVFS which reduces the operating frequency of the processor cores to reduce dynamic power consumption without degrading the application performance significantly (Hsu and Feng 2005).

GAMESS (Schmidt, Baldridge, Boatz, Elbert, Gordon, Jensen, Koseki, Matsunaga, Nguyen, Su, Windus, Dupuis, and Montgomery 1993) is one of the most representative freely available quantum chemistry applications used worldwide to do ab initio electronic structure calculations. A wide range of quantum chemistry computations may be accomplished using GAMESS, ranging from basic Hartree-Fock and Density Functional Theory computations to high-accuracy multi-reference and coupled-cluster computations. For the experiments conducted in this work, an input file was constructed which describes a GAMESS calculation on 20 water molecules using the 3rd-Order Fragment Molecular Orbital (FMO3) method at the MP2/6$31 \mathrm{G}(\mathrm{d}, \mathrm{p})$ level of theory. The calculation uses the standard DDI (direct data interface) (R.M. Olson, Gordon, and Rendell 2003) implementation in GAMESS As such, each fragment N-mer (monomer, dimer, and trimer) is calculated sequentially using all compute elements allocated to the executable. 3-body calculations at the RHF/6-31G(d,p) (Restricted Hartree-Fock) level of theory are also performed and are critical in order to capture the significant exchange and charge-transfer effects present in a cluster of water molecules. The input is referred to as wat-20 in the rest of the paper.

In this work, two hardware platforms operating DDR4 and DDR3 chips were selected and then the differing memory intensity of the same application along with resulting efficacy of DVFS on these two platforms was explored. Various system and software parameters, such as hardware prefetching, and compiler options were tweaked in order to better understand the relative behavior of memory intensity on the two platforms. Experiments depicted that, for the same application, DDR4 provided more room to apply DVFS whereas the theoretical performance models suggested otherwise. Hence, this unexpected effect of memory intensity on DDR4 is investigated here by comparison with that on DDR3, while aiming to later calibrate the performance models proposed by the authors in their previous work (Sundriyal and Sosonkina 2016).

The rest of the paper is organized as follows. Section 2 compares the salient features of the DDR3 and DDR4 chips used in this work. Section 3 explores the proposed performance models to theoretically establish the improvement in performance of an application when moving from DDR3 to DDR4 platform, given the PE (processing element) performance remains the same. Section 4 studies the effect of modifying various system and software parameters on the application memory intensity on both the DDR3 and DDR4 platforms. Section 5 and Section 6 provide related work and conclusions, respectively.

\section{DDR3 VS DDR4}

The first comparison between DDR3 and DDR4 is from the perspective of power consumption. DDR4 operates at a lower supply voltage (1.2v) compared to DDR3 (1.5v) and also implements an algorithm to enter a "standby" mode more frequently than DDR3 to save power. The other important improvement of DDR4 over DDR3 is better bandwidth and memory latency. The prefetch length which is the number of bits of data per clock cycle transferred from the memory array to the memory internal I/O (input/output) buffer, remains the same in both DDR4 and DDR3 but bank capacity and overall memory density is higher in DDR4 compared to DDR3 (Poole 2018).

Table 1 depicts the two hardware platforms, Borges and Gwent, used in this work for the experimental evaluation. Borges is a dual socket platform which consists of two Xeon E5-2650 8 core Sandy-Bridge EP processors with $64 \mathrm{~GB}$ of DDR3. Gwent, on the other hand, comprises two Xeon E5-2650 v3 10 core processors with $32 \mathrm{~GB}$ of DDR4 memory. 
Table 1: System parameters of the two platforms used in this work.

\begin{tabular}{|c|c|c|}
\hline & Borges & Gwent \\
\hline Processor & Intel(R) Xeon(R) CPU E5-2650 & Intel(R) Xeon(R) CPU E5-2650 v3 \\
\hline Memory Type & DDR3 & DDR4 \\
\hline Memory Capacity & $64 \mathrm{~GB}$ & $32 \mathrm{~GB}$ \\
\hline Core Frequency Range & $1.2-2 \mathrm{GHz}$ & $1.2-2.3 \mathrm{GHz}$ \\
\hline
\end{tabular}

Table 2: Latency and bandwidth of the DDR3 and DDR4 platforms measured in this work.

\begin{tabular}{|c|c|c|}
\hline & Latency (ns) & Bandwidth (MB/s) \\
\hline Borges & 155 & 42908 \\
\hline Gwent & 80 & 60142 \\
\hline
\end{tabular}

Table 2 provides the latency and peak bandwidth measured through the lat_mem_rd utility in the lmbench (McVoy and Staelin 1996) suite and Intel memory latency checker (Vishwanathan 2017), respectively for the Gwent and Borges platforms. DDR4 improves the latency and bandwidth over DDR3 by $48 \%$ and $40 \%$, respectively.

\section{PERFORMANCE MODEL}

The execution time of a program can be divided into two separate parts, on-chip time $t_{o n}$ and off-chip time $t_{o f f}$, such that $t_{o n}$ and $t_{o f f}$ are non-overlapping (Choi, Soma, and Pedram 2005). The time $t_{o f f}$ consists of stall cycles, such as memory, I/O, branch misprediction, and reservation station stalls, during which the PE is not doing any useful work. DVFS only scales the on-chip time of an application whereas the off-chip time largely remains unaffected. Therefore, the ratio of the off-chip and on-chip time in an application primarily decides the room for application of frequency scaling.

$$
\mu \tau(i)=\frac{f_{p}(i)}{\mathrm{CPM}_{\text {exe }}+\alpha \times \mathrm{MAPM} \times \beta \times \frac{f_{p}(i)}{f_{p}(1)}} .
$$

Eq. (1) shows the basic performance model proposed and verified in a previous work (Sundriyal and Sosonkina 2016) where

- $\quad \mu \tau(i)$ is the actual number of micro-operations retired per second at processor frequency $f_{p}(i)$.

- $\quad \mathrm{CPM}_{\text {exe }}$ is the number of cycles per micro-operations retired barring the memory accesses in a second.

- $\alpha(0 \leq \alpha \leq 1)$ is the OOO overlap factor, which determines the extent of memory stalls overlapped with execution cycles.

- $\quad$ MAPM is the number of memory accesses per micro operation retired in a second.

- $\quad \beta$ is the number of cycles corresponding to the memory-access latency.

It can be observed from Eq. (1) that as there is an increase in the memory intensity (MAPM) and the memory access latency $(\beta)$ (which correspond to the off-chip time) for a given application on a platform and rest of the variables remaining constant, the denominator increases and subsequently the application performance (micro-operations retired) degrades. As DDR4 has substantially better bandwidth and latency compared to DDR3, it can be expected that a given application would have lower or at least similar memory intensity on DDR4 compared to DDR3, given the other parameters in Eq. (1) remain the same.

Table 3 shows the memory intensity of different configurations of the NAS CG benchmark on the Gwent and Borges platforms. The NAS CG benchmark has been depicted as "xx.yy.zz", where "xx", "yy", and 
Table 3: Memory intensity of CG benchmark on Gwent and Borges measured in memory accesses per micro-operation retired per second.

\begin{tabular}{|c|c|c|}
\hline Benchmark & Gwent & Borges \\
\hline CG.C.1 & 0.005 & 0.000009 \\
\hline CG.C.16 & 0.0038 & 0.001170 \\
\hline
\end{tabular}

Table 4: Energy delay product (EDP) on DDR4 platfom, normalized to the one on DDR3 for each of the three inputs.

\begin{tabular}{|c|c|}
\hline Benchmark & Normalized EDP \\
\hline CG.C.1 & 0.21 \\
\hline CG.C.16 & 0.15 \\
\hline wat-20 & 0.225 \\
\hline
\end{tabular}

"zz" denote benchmark name, class, and number of processes used, respectively. The NAS CG benchmark was chosen for the experiments since it depicted a near uniform memory intensity throughout its execution irrespective of the underlying hardware platform. To keep the parameters and execution scenario near similar on both the platforms in accordance with Eq. (1), both platforms were operated at a processor frequency of $2.0 \mathrm{GHz}$ and MVAPICH 2.0 was used. It can be observed from Table 3 that the memory intensity of the CG benchmark increases drastically in both the configurations when moving from DDR3 (Borges) to DDR4 (Gwent) platform. In the following sections, different system and hardware parameters are tweaked on both the platforms to determine the effect on the benchmark memory intensity. Also, this could help gain insight into the relative difference of benchmark memory intensity on DDR3 and DDR4 platforms.

\subsection{Energy Delay Product}

An analysis of the relative memory intensity of the two platforms prompts a a comparison of their energy efficiencies. Table 4 depicts the energy delay product (EDP) for the DDR4 (Gwent) platform, normalized to the EDP on the DDR3 (Borges) platform for the three inputs. It can be observed that the DDR4 platform provides substantially better energy efficiency by reducing here the EDP on average by $\sim 80 \%$. This improvement in energy efficiency is provided not only by the overall improvement in execution time but also by a reduction in power consumption by nearly $2.5 x$ on the DDR4 over the DDR3 platform.

\section{SYSTEM AND HARDWARE PARAMETER MODIFICATION}

The memory intensity of an application is dependent on many system software and hardware managed parameters. In this section, three such parameters were selected because they are known to potentially affect the memory intensity the most. Next they were changed to determine the effect on the memory intensity of NAS CG benchmark on the two hardware platforms. Specifically, these parameters are compiler optimizations, prefetching, and rank/channel interleaving (i.e., spreading data across channels/ranks to increase read bandwidth). Experimental results provide deeper insights into the extent to which the off-chip time of an application is affected. Moreover, the experiments also help in understanding that how the memory intensity is affected and whether or not these parameters are responsible for the increased memory intensity on DDR4 over DDR3. 
Table 5: Memory intensity and off-chip to on-chip time ratio of the NAS CG benchmark and GAMESS FMO input with the four prefetchers enabled (E) and disabled (D) on the two hardware platforms.

\begin{tabular}{|c|c|c|c|c|c|c|c|c|}
\hline Benchmark & \multicolumn{3}{|c|}{ Gwent } & \multicolumn{3}{c|}{ Borges } \\
\hline & \multicolumn{2}{|c|}{ E } & \multicolumn{2}{c|}{ D } & \multicolumn{2}{c|}{ E } & \multicolumn{2}{c|}{ D } \\
\hline & MAPM & $\frac{t_{\text {off }}}{t_{o n}}$ & MAPM & $\frac{t_{\text {off }}}{t_{o n}}$ & MAPM & $\frac{t_{\text {off }}}{t_{o n}}$ & MAPM & $\frac{t_{\text {off }}}{t_{o n}}$ \\
\hline CG.C.1 & 0.00936 & 1.965 & 0.020 & 2.47 & 0.000008 & 0.001 & 0.000016 & 0.01 \\
\hline CG.C.16 & 0.003884 & 3.8 & 0.0166 & 3.8 & 0.00117 & 0.273 & 0.021 & 1.3 \\
\hline wat-20 & 0.000016 & 0.08 & 0.0003 & 0.11 & 0.000018 & 0.45 & 0.0000012 & 0.03 \\
\hline
\end{tabular}

\subsection{Compiler Memory Optimizations}

Compiler memory optimizations can fundamentally determine where an object exists and when it can be accessed in the main memory (David 2019). By reordering computation and data layout optimizations, the compiler attempts to determine the time when an object will be accessed along with the address where it will reside to improve performance. Examples of such optimizations include loop interchange, cache blocking and skewing. The compiler optimizations can alter the memory access pattern of an application.

To test whether the compiler optimizations are causing the apparent difference in memory intensity between DDR3 and DDR4, the optimizations were turned off by using the "-O0" flag. Moreover, similar versions of gcc (4.8) and gfortran (4.8) were used on both the hardware platforms so that any potential issues that might arise due to different versions of compilers can be avoided.

Experiments conducted on Gwent and Borges using the CG.C.1 and CG.C.16 benchmarks depicted no difference in memory intensity for the two benchmarks on the same platform. Moreover, no difference in execution time as well was observed for the two variants of the CG benchmark. Therefore, it can be concluded that compiler induced optimizations are indeed not responsible for the difference in memory intensity of the CG benchmark between the two platforms.

\subsection{Prefetching}

Prefetching in processors is a strategy under which adjacent data apart from the data in demand is fetched from memory to exploit spatial locality and improve performance. Prefetching can be directed either through the software (compiler/programmer) or the hardware. Since all the compiler optimizations can be turned off by using the "-O0" flag, the focus is on hardware prefetching which is managed through the BIOS in modern computing systems. More specifically, four types of hardware prefetching mechanisms are used in the hardware platforms employed in this work:

- $\quad$ MLC Streamer: Memory Latency Checker (MLC) Streamer prefetcher is a speculative prefetch unit within the processor.

- MLC Spatial: This prefetcher when enabled, fetches adjacent cache line when required data is not currently in the cache.

- DCU Streamer: The data cache unit (DCU) prefetcher fetches the next cache line into L1 data cache from L2 or system memory during unused cycles if it sees that the processor core has accessed several bytes sequentially in a cache line as data.

- DCU IP: The instruction prefetcher (IP) when enabled, fetches the next cache line into L1 instruction cache from L2 or system memory during unused cycles if it sees that the processor core has accessed several bytes sequentially in a cache line as data 
Table 6: MAPM and the off-chip to on-chip time ratio for the selected applications with varying channel interleaving and fixed rank interleaving on Borges.

\begin{tabular}{|c|c|c|c|c|}
\hline Benchmark & \multicolumn{4}{|c|}{ Borges } \\
\hline & \multicolumn{2}{|c|}{$(2,1)$} & \multicolumn{2}{c|}{$(4,1)$} \\
\hline & MAPM & $\frac{t_{\text {off }}}{t_{\text {on }}}$ & MAPM & $\frac{t_{\text {off }}}{t_{\text {on }}}$ \\
\hline CG.C.1 & 0.000001 & 0.09 & 0.000001 & 0.08 \\
\hline CG.C.16 & 0.00008 & 1.30 & 0.000097 & 0.58 \\
\hline wat-20 & 0.000007 & 0.124 & 0.00008 & 0.14 \\
\hline
\end{tabular}

Table 7: MAPM and the off-chip to on-chip time ratio for the selected applications with fixed channel interleaving and varying rank interleaving on Borges.

\begin{tabular}{|c|c|c|c|c|}
\hline Benchmark & \multicolumn{4}{|c|}{ Borges } \\
\hline & \multicolumn{2}{|c|}{$(1,2)$} & \multicolumn{2}{c|}{$(1,4)$} \\
\hline & MAPM & $\frac{t_{\text {off }}}{t_{\text {on }}}$ & MAPM & $\frac{t_{\text {off }}}{t_{o n}}$ \\
\hline CG.C.1 & 0.000001 & 0.007 & 0.000001 & 0.006 \\
\hline CG.C.16 & 0.000007 & 1.22 & 0.000007 & 1.23 \\
\hline wat-20 & 0.000006 & 0.000003 & 0.000011 & 0.0001 \\
\hline
\end{tabular}

Table 5 depicts the comparison of memory intensity and the off-chip to on-chip time ratio of the NAS CG benchmark and the wat-20 input on the two hardware platforms for the four prefetchers enabled and disabled, denoted as "E" and "D", respectively. The memory intensity values were obtained by determining the ratio of the number of L3 cache misses to the number of micro-operations retired. The ratio of off-chip to on-chip time was calculated for an application by executing an application at different core frequencies and then using a regression analysis proposed in (Sundriyal, Sosonkina, Liu, and Schmidt 2011). It can be observed that on both the platforms, disabling the prefetchers increases the memory intensity substantially for the NAS CG benchmark. This is expected and is due to the fact that with the prefetchers disabled, the spatial locality associated with the execution is not exploited and processor has to access memory much more frequently compared to the case where adjacent data is already prefetched. Subsequently, substantial performance degradation was also observed when prefetchers were disabled. More specifically, performance degradation of up to $17 \%$ was observed when prefetching was disabled. On the other hand, for the wat -20 input on Borges, performance improvement of $23 \%$ was observed along with reduction in memory intensity when prefetching was disabled on Borges and this anomalous behavior is currently being investigated.

Regarding the effect of shutting off the four prefetchers separately, individually disabling the first three prefetchers namely MLC Streamer, MLC Spatial and DCU Streamer did not affect the performance and memory intensity of both the NAS CG benchmark and the wat-20 input. Only individually shutting off the DCU IP prefetcher degraded performance of the CG.C.1 benchmark by nearly $70 \%$ on Borges. In general, it can be concluded that the disabling prefetching mechanism increases in degraded performance and increased memory intensity (and the room for application of DVFS). Moreover, it is also not responsible for the difference in the memory intensity on the two hardware platforms.

\subsection{Channel and Rank Interleaving}

Memory devices are organized internally as ranks, banks and channels (Jacob, Ng, and Wang 2007). At the base level, memory devices are made up of multiple arrays of single-bit storage cells arranged in a two-dimensional structure formed by the intersection of individual rows (Word Lines) and columns (Bit Lines). These structures are called banks and when associated in groups eight (DDR3) or sixteen (DDR4), 
Table 8: MAPM and the off-chip to on-chip time for the selected applications with varying channel and rank interleaving on Borges.

\begin{tabular}{|c|c|c|c|c|}
\hline Benchmark & \multicolumn{4}{|c|}{ Borges } \\
\hline & \multicolumn{2}{|c|}{$(1,1)$} & \multicolumn{2}{c|}{$(4,8)$} \\
\hline & MAPM & $\frac{t_{\text {off }}}{t_{\text {on }}}$ & MAPM & $\frac{t_{\text {off }}}{t_{\text {on }}}$ \\
\hline CG.C.1 & 0.000001 & 0.08 & 0.000001 & 0.071 \\
\hline CG.C.16 & 0.000007 & 1.27 & 0.000135 & 0.053 \\
\hline wat-20 & 0.000009 & 0.003 & 0.000011 & 0.0003 \\
\hline
\end{tabular}

these banks form the next higher logical unit, known as a rank. A channel can be defined as a dedicated path of communication between processor and memory. Having multiple channels provides higher memory bandwidth by multiplying the data rate and the number of channels present. The Xeon processors used in this processor support quad-channel memory configuration.

Storing data across different memory channels allows the processor to utilize its multiple memory controllers. When all four channels are populated with data, the processor interleaves memory accesses across the multiple memory channels which improves the throughput. Rank interleaving stores data across different ranks of a DIMM. Since a minimum of two ranks are required for rank interleaving to be supported, double-sided DIMMs are required to enable this feature. In the platforms used in this work, a maximum of 8 -way rank interleaving is supported.

Table 6 depicts the MAPM values and off-chip to on-chip time ratio of the NAS CG benchmark and wat -20 input with varying level of channel interleaving and fixed level of rank interleaving on Borges. Since Gwent did not have any options for modifying channel/rank interleaving in the BIOS, the relevant experiments could not be performed. The interleaving schemes are depicted as $(a, b)$ where " $a$ " and " $b$ " stand for channel and rank interleaving modes, respectively.

It can be observed from Table 6 that for the CG.C.1 benchmark, there isn't much change in MAPM when level of channel interleaving is varied, keeping the level of rank interleaving fixed. Same can be said for the CG.C.16 benchmark except the ratio of off-chip to on-chip time is nearly halved when level of channel interleaving is increased. The wat-20 input remains consistent in terms of memory intensity with the change in the level of channel interleaving. In terms of application performance, no visible change was observed in the execution time of the three benchmarks.

In Table 7, where the level of channel interleaving is kept constant and level of rank interleaving is varied, no substantial changes for the three benchmarks in terms of their memory intensity were observed. On the other hand, significant performance degradation ( 68\%) was observed for the CG.C.16 benchmark when level of rank interleaving was increased, keeping the channel interleaving constant. This performance degradation could be attributed to the scenario where the processor has to request data from different ranks which can be multiple DIMMs using a single channel, possibly resulting in increased memory access latency.

Table 8 shows the memory intensity of the selected benchmarks, for the case where both the levels of channel and rank interleaving were varied. The interleaving scheme $(1,1)$ for the CG.C.1 benchmark as shown in Table 8 is significantly compute intensive with negligible off-chip time compared to the on-chip time. When the interleaving scheme is changed to $(4,8)$, the MAPM value and the compute intensity do not change by much for the CG.C.1 benchmark. As for the CG.C.16 benchmark, in the default $(1,1)$ configuration, it is substantially memory intensive with off-chip time being greater than on-chip time. But when the interleaving configuration is changed to $(4,8)$, it turns compute intensive due to the improved channel bandwidth. In the case of the wat -20 input, no substantial changes in memory intensity were observed while moving from $(1,1)$ to $(4,8)$ interleaving configuration. 
Sundriyal, Westheimer and Sosonkina

\section{RELATED WORK}

It has been observed that memory stalls tend to be most significant in terms of blocking the instruction flow (Zhang and Chang 2014) out of all the stalls. Therefore, the phenomena of limiting system performance due to the difference in processor speed and memory access latency has been termed as memory wall (Wulf and McKee 1994). Power is one of the most important design constraints for the next generation of computing systems forcing the research community to continuously evaluate and redefine the objectives of HPC power management. There have been two general approaches to obtaining energy savings during parallel application execution. The first approach is to focus on identifying stalls during execution by measuring architectural parameters from performance counters as proposed in $(\mathrm{Ge}$, Feng, Feng, and Cameron 2007, Hsu and Feng 2005, Huang and Feng 2009). The second approach determines the communication phases to apply DVFS as, for example, in (Lim, Freeh, and Lowenthal 2006) and (Freeh and Lowenthal 2005). Since many applications may not exhibit communication phase behavior and do not not provide enough room for DVFS, energy saving strategies based on determining stalls are more reliable.

The clock duty cycle distortion (DCD) in DDR3 and DDR4 were compared in (Kim, Feng, Mokhtaari, Adhyaru, Shaikh, Natarajan, and Oh 2016) and it was concluded that DCD was a function of many parameters such as number of physical channels and the operating frequency. In (Bhati, Chang, Chishti, Lu, and Jacob 2016), refresh command sequence, penalties associated with the refresh operation and performance tradeoffs for different DDR devices were studied. Authors in (Zhang and Guo 2017) propose a novel fine-grained DRAM architecture which interleaves data from different banks on the global I/O to improve performance. This work differs from the previous research in specifically determining the DVFS efficacy on the DDR4 and DDR3 platforms and determining the change in memory intensity on these platforms when certain software and hardware parameters were modified.

\section{CONCLUSIONS AND FUTURE WORK}

In this paper, a study was performed where the memory intensity of a chosen set of applications was examined on DDR3 and DDR4 based platforms. It was observed that the memory intensity of applications depicted a substantial increase while moving from DDR3 to DDR4, despite predictions suggesting otherwise based on a verified performance model. Next, certain software and hardware parameters (compiler optimizations, prefetching and channel/rank interleaving) were chosen and their values were tweaked to examine the effect on memory intensity of the two platforms chosen. It was concluded that those parameters did not contribute to the increased memory intensity on DDR4 compared to DDR3. Moreover, modifying these parameters in some cases did change the memory intensity substantially for the selected set of applications thereby affecting the room for application of processor frequency scaling.

Future work will focus on delving deeper into the selected parameters and how simultaneously modifying those could affect the memory intensity and the application performance overall. Other hardware parameters, such as DRAM timing parameters and QPI (quick path interconnect) bandwidth will also be included in the study. Efforts will be guided towards enhancing the performance model studied in this work to possibly include the effects of modifying these parameters.

\section{ACKNOWLEDGMENTS}

This work was supported in part by the U.S. Department of Energy (DOE) Office of Science, Office of Basic Energy Sciences, Computational Chemical Sciences (CCS) Research Program under work proposal number AL-18-380-057 and the Exascale Computing Project (ECP) through the Ames Laboratory, operated by Iowa State University under contract No. DE-AC00-07CH11358, by the U.S. Department of Defense High Performance Computing Modernization Program, through a HASI grant. 


\section{REFERENCES}

Bhati, I., M. Chang, Z. Chishti, S. Lu, and B. Jacob. 2016, Jan. "DRAM Refresh Mechanisms, Penalties, and Trade-Offs". IEEE Transactions on Computers vol. 65 (1), pp. 108-121.

Choi, K., R. Soma, and M. Pedram. 2005. "Fine-grained dynamic voltage and frequency scaling for precise energy and performance tradeoff based on the ratio of off-chip access to on-chip computation times". Computer-Aided Design of Integrated Circuits and Systems, IEEE Transactions on vol. 24 (1), pp. 18 28.

M. David January 2019. "Putting Your Data and Code in Order". https://software.intel.com/en-us/articles/ putting-your-data-and-code-in-order-optimization-and-memory-part-1. [Online; accessed 1-January2019].

Freeh, V., and D. Lowenthal. 2005. "Using multiple energy gears in MPI programs on a power-scalable cluster". In Proceedings of the tenth ACM SIGPLAN symposium on Principles and practice of parallel programming, pp. 164-173.

Ge, R., X. Feng, W. Feng, and K. Cameron. 2007, Sep.. "CPU MISER: A Performance-Directed, Run-Time System for Power-Aware Clusters". In Parallel Processing, 2007. ICPP 2007. International Conference on, pp. 18.

Ge, R., X. Feng, S. Song, H. Chang, D. Li, and K. Cameron. 2010. "PowerPack: Energy Profiling and Analysis of High-Performance Systems and Applications". Parallel and Distributed Systems, IEEE Transactions on vol. 21, pp. 658-671.

Hsu, C., and W. Feng. 2005, Nov.. "A Power-Aware Run-Time System for High-Performance Computing". In Supercomputing, 2005. Proceedings of the ACM/IEEE SC 2005 Conference, pp. 1.

Huang, S., and W. Feng. 2009, May. "Energy-Efficient Cluster Computing via Accurate Workload Characterization". In Cluster Computing and the Grid, 2009. CCGRID'09. 9th IEEE/ACM International Symposium on, pp. 68-75.

Jacob, B., S. Ng, and D. Wang. 2007. Memory Systems: Cache, DRAM, Disk. San Francisco, CA, USA, Morgan Kaufmann Publishers Inc.

JEDEC January 2019a. "DDR3 Standard, JESD79-3F". https://www.jedec.org/sites/default/files/docs/ JESD79-3F.pdf. [Online; accessed 3-January-2019].

JEDEC January 2019b. "DDR4 Standard, JESD79-4". https://www.jedec.org/sites/default/files/docs/ JESD79-4.pdf. [Online; accessed 3-January-2019].

Kim, G., J. Feng, M. Mokhtaari, J. Adhyaru, R. Shaikh, B. Natarajan, and D. Oh. 2016, Fourth. "Analysis and comparison of DDR3/DDR4 clock duty-cycle-distortion (DCD) for UDIMM and discrete SDRAM component configurations". IEEE Electromagnetic Compatibility Magazine vol. 5 (4), pp. 133-139.

Lim, M., V. Freeh, and D. Lowenthal. 2006. "Adaptive, transparent frequency and voltage scaling of communication phases in MPI programs". In Proceedings of the 2006 ACM/IEEE conference on Supercomputing.

McVoy, L., and C. Staelin. 1996. "Lmbench: Portable Tools for Performance Analysis". In Proceedings of the 1996 Annual Conference on USENIX Annual Technical Conference, ATEC '96, pp. 23-23. Berkeley, CA, USA, USENIX Association.

Ian Poole December 2018. "DDR4 Memory". https://www.radio-electronics.com/info/data/semicond/ memory/sdram-ddr4-memory.php. [Online; accessed 20-December-2018].

R.M. Olson, M. S., M. Gordon, and A. Rendell. 2003, Nov.. "Enabling the Efficient Use of SMP Clusters: The GAMESS/DDI Model”. In Supercomputing, 2003 ACM/IEEE Conference, pp. 41. 
Schmidt, M. W., K. Baldridge, J. Boatz, S. Elbert, M. Gordon, J. Jensen, S. Koseki, N. Matsunaga, K. Nguyen, S. Su, T. Windus, M. Dupuis, and J. J. Montgomery. 1993, Nov.. "General atomic and molecular electronic structure system". J. Comput. Chem. vol. 14, pp. 1347-1363.

Sundriyal, V., and M. Sosonkina. 2016. "Joint Frequency Scaling of Processor and DRAM". The Journal of Supercomputing vol. 72 (4), pp. 1549-1569.

Sundriyal, V., M. Sosonkina, F. Liu, and M. Schmidt. 2011. "Dynamic Frequency Scaling and Energy Saving in Quantum Chemistry Applications". In Proceedings of the 2011 IEEE International Symposium on Parallel and Distributed Processing Workshops and PhD Forum, IPDPSW '11, pp. 837-845. Washington, DC, USA, IEEE Computer Society.

V. Vishwanathan July 2017. "Intel Memory Latency Checker". https://software.intel.com/en-us/articles/ intelr-memory-latency-checker. [Online; accessed 27-November-2017].

Wulf, W., and S. A. McKee. 1994. "Hitting the Memory Wall: Implications of the Obvious". Technical report, Charlottesville, VA, USA.

Zhang, C., and X. Guo. 2017, July. "Enabling efficient fine-grained DRAM activations with interleaved I/O”. In 2017 IEEE/ACM International Symposium on Low Power Electronics and Design (ISLPED), pp. 1-6.

Zhang, Z., and J. M. Chang. 2014, May. "A Cool Scheduler for Multi-Core Systems Exploiting Program Phases". IEEE Transactions on Computers vol. 63 (5), pp. 1061-1073.

\section{AUTHOR BIOGRAPHIES}

VAIBHAV SUNDRIYAL is currently working as a research scientist at Old Dominion University Research Foundation. His email is vsundriy@ odu.edu.

MASHA SOSONKINA is a Professor in Department of Modeling, Simulation and Visualization engineering at Old Dominion University. Her email is msosonki@odu.edu.

BRYCE WESTHEIMER is a graduate student in Department of Chemistry at Iowa State University. His email is westheb@iastate.edu. 